\title{
A Principled Analysis of the Interrelation Between Vehicular Communication and Reasoning Capabilities of Autonomous Vehicles
}

\author{
Mauro Vallati and Lukáš Chrpa
}

\begin{abstract}
The current growth in urbanisation is posing serious problems in urban areas worldwide. Traditional traffic control tools, such as SCOOT and SCATS, are widely exploited in major cities, but given the increasing traffic demands, they need to be complemented with additional mechanisms. Within this context, the emerging interest in autonomous vehicles (AVs) points to the direction of a paradigm shift in the way in which traffic is controlled and managed. This is due to the fact that AVs can exploit different types of communication, hence take better informed decisions. Despite the amount of work dedicated to engineering solutions for supporting and implementing the different types of vehicular communication, there is a lack of analysis focusing on the implications of exploiting one (or more) type of communication.

In this work, focusing on urban areas, we provide a principled and detailed analysis of the impact of different kinds of communication on reasoning capabilities of vehicles and of urban traffic control (e.g. level of deliberation). The outcome of the performed analysis can then be fruitfully exploited by experts to better understand and support communication and reasoning, according to the needs of the controlled areas.
\end{abstract}

\section{INTRODUCTION}

In recent years, there has been a huge effort in the development of autonomous vehicles (AVs), which are capable of driving autonomously. Autonomous vehicles are able to effectively and efficiently reason on available data in order to increase traffic safety, fuel efficiency, and comfort for passengers, and to lead to a better use of available infrastructure. $\mathrm{AVs}$ therefore point to the direction of a paradigm shift in the way vehicles behave and interact, particularly in urban areas. A pivotal aspect of AVs, that would allow to unleash their full potential, resides in the way in which they can be connected with other vehicles or with the urban infrastructure, in order to share information and being able to take better informed decisions. Typical typologies of vehicular communications include vehicle-to-vehicle [1], vehicle.to-objects [2], [3], and vehicle-to-infrastructure [4], or a mix of them [5]. According to the type of communication, AVs can use Vehicular Adhoc NETworks (VANETs), or exploiting existing networks and architectures, such as GPS or cellular networks. There is a very active strand of research focusing on the actual technologies to be used for implementing the communication, and to minimise the requirements of additional investments from traffic authorities.

With an expected growth of urban population to $66 \%$ by 2050 [6], traffic volume in urban areas might witness

M. Vallati is with School of Computing \& Engineering, University of Huddersfield, Huddersfield, UK.

L. Chrpa is with Faculty of Mathematics and Physics at Charles University and Artificial Intelligence Center at Czech Technical University, Prague, CZ. a dramatic increase with respect to current traffic situation. Consequently, traffic congestion will worsen; also because of its economical, health, and environmental repercussions [7]. Traditional urban traffic control (UTC) techniques such as SCOOT [8], MOVA [9], and SCATS [10], which are deployed all over the world, adapt their own green phase lengths depending on current traffic conditions. Such adjustment to "fixed plans" can be made in rush hours in order to cope with increased traffic on main roads. However, with expected increase of traffic in the following decades and with expected deployment of AVs, there is a need to employ "smarter" techniques for UTC that utilise and exploit additional capabilities of AVs.

Despite the amount of work dedicated to engineering solutions for supporting and implementing the different types of vehicular communication, there is a lack of analysis focusing on the implications of exploiting one (or more) type of communication. As it is apparent, the sort of data and the kind of interactions made available by the communication can lead to very different goals to be dealt with. For instance, different types of communication support a different type of reasoning, from reactive to deliberative.

In this work, focusing on urban areas, we provide a principled and detailed analysis of the impact of different kinds of communication on vehicles and on UTC. The analysis can then be fruitfully exploited by experts to better understand, support, and combine different types of communications according to needs and resources of the controlled areas. The analysis is based on a set of criteria, taking into account aspects such as the knowledge available to each involved entity, the set of goals that can be aimed at according to the available data, and the computational and infrastructural requirements needed. Our analysis allows to gain a more accurate overview of the aims and objectives of each type of vehicular communication, and point to the implications and effects that such decisions have on the capabilities of vehicles and traffic controllers. Furthermore, we qualitatively discuss the importance of penetration rate in order to gain noticeable benefits from the exchange of information.

\section{Categories of Vehicular Communications}

This section is devoted to describe and categorise vehicular communications considered in this work. Notably, we focus on fully autonomous vehicles, where no human intervention is required in order to drive the vehicle.

1) No communication. This category includes $A V s$ that do not explicitly and actively communicate and share information with any other entity. Vehicles in this 
category can be seen as the extension of currently available vehicles, with the addition of autonomous driving capability and sensing. In fact, most of the autonomous vehicle in development are based on a perception system consisting of onboard sensors, allowing the vehicle to create a map of the surroundings and control the motion [11].

From the traffic control perspective, information about vehicles and traffic can be collected via existing sensors (such as cameras, bluetooth, etc.) on the network. The vehicle may decide the best route to take by exploiting a SatNav system that is connected to a cellular network, and has information about the traffic, but the vehicle is not actively engaging in the communication.

2) Vehicle-to-vehicle (V2V). This class incorporates approaches and techniques that allows to perform communication among vehicles. It allows vehicles to establish a set of ad-hoc networks to cooperate, in order to share information and increase safety and efficiency. Communication can be 1-to-1, or can be among a group of vehicles, identified by proximity. This sort of communication, when established between a group of vehicles, allows the group to cooperate in order to perform complex manoeuvres.

Vehicles in this class can still exploit information gained by using connected SatNavs, as for those in the no communication category, but can not directly communicate with infrastructure objects.

3) Vehicle-to-objects (V2X). Vehicles able to perform V2X communications encompass the abilities of those in the $\mathrm{V} 2 \mathrm{~V}$ category. $\mathrm{V} 2 \mathrm{X}$ allows to communicate with other vehicles, as well as with part of the local infrastructure. For instance, a vehicle can communicate with the traffic light on the next junction in order to obtain green light optimal speed advisory [12] -i.e., the speed that maximises the probability to find the traffic light on green by the time the vehicle will arrive at the junction, thus reducing pollution due to queuing-. In V2X, we consider a 2-way communication to be established: the $X$ object should be able to exchange messages and information with nearby AVs.

It should be noted that the V2X category we consider in this work, is thought for decentralised reasoning and traffic control: there is no exchange of information with a central controller that has a global view of the network conditions. Instead, all information and reasoning is on local basis. However, as for the $\mathrm{V} 2 \mathrm{~V}$ category, also V2X vehicles can exploit the benefits of SatNavs, and are still providing information to centralised traditional UTC systems via surface sensors.

4) Centralised infrastructure-to-vehicle (I2V, V2I). This class covers approaches where the vehicle can communicate (a 2-way communication, so it can handle responses and instructions) with the centralised control centre that is in charge of performing traffic control for the current urban area. In a broader sense, $\mathrm{I} 2 \mathrm{~V}$ points to the direction of an enhanced traffic control system, that can coordinate and collaborate with AVs navigating in the region.

For the sake of this analysis, we consider vehicles in this class not able to perform $\mathrm{V} 2 \mathrm{~V}$ or $\mathrm{V} 2 \mathrm{X}$ communications: this allows us to provide a more focused discussion about the different strengths and weaknesses of the communication categories. It should be noted, however, that in $\mathrm{I} 2 \mathrm{~V}$ vehicles are still providing, beside information sent via direct communication, information via sensors distributed in the network. Given the ability to communicate with the centralised controller, AVs do not need to rely on SatNavs, as routing information -whether needed- are provided by the urban traffic controller.

\section{CRITERIA}

In order to provide a principled and coherent approach for assessing and comparing the different vehicular communication categories, here we rely on a number of criteria. In a nutshell, a criterion represents a particular aspect or perspective, and their exploitation gives the possibility to investigate communication categories from different angles, and to provide a focused yet multi faceted analysis.

The following criteria are considered in this work.

- Knowledge and Reasoning. Here we focus on assessing what sort of information and data are available and can be exchanged by the category of vehicular communication. Does the vehicle (infrastructure) have a global or a local view of the network condition? What sort of reasoning can be performed? Are there strict limits in terms of available computational resources?

- Achievable Goals. This aspect includes the sort of high level goals that, through the specific type of communication and the available data, can be described and (hopefully) achieved.

- Infrastructural Requirements. This criterion focuses on aspects related to the infrastructure that is needed in order to sustain a given type of communication. In a broader sense, infrastructure include the set of protocols needed, the hardware devices required by vehicles and that should be put in place in the network surface, as well as sensors, etc.

- Communication Requirements. This aspect considers elements that are related to requirements of the communication, such as latency or sensitivity to errors or corrupt information, and security and privacy of the vehicle and passengers. The importance of timely and reliable communication is also considered by this criterion. Finally, the usage of the network, in terms of the amount of information to be shared with involved (and with non-involved) vehicles, is also considered.

- Importance of Penetration Rate. In this context, penetration rate indicates the percentage of vehicles that are exploiting the specific type of communication. How dependent are the benefits of communication on the penetration rate? Are benefits proportional to penetration rate? 


\section{ANALYSIS}

In this section all the vehicular communication categories introduced in this paper are discussed and evaluated against the outlined criteria.

\section{A. No Communication}

This category assumes that no 2-way communication is established by the vehicle with the infrastructure or with other vehicles.

1) Knowledge and Reasoning: By making the reasonable assumption that every $\mathrm{AV}$ is equipped with a SatNav that is connected to Internet [13], each vehicle has therefore available knowledge about the current status of the urban network, at least in terms of congestion level of links. Such knowledge can be exploited by the vehicle for identifying best routes for reaching the desired destination.

Further knowledge is constantly acquired by the vehicle's sensors. Such knowledge, however, can only by exploited for driving purposes as it refers only to the immediate surroundings of the vehicle and allows to provide a map of the environment.

2) Achievable Goals: In terms of achievable goals, two main perspectives have to be taken into account: the vehicle and the urban controller perspectives.

The vehicle aims at maximising the safety for passengers, by sensing the surroundings, while controlling longitudinal and latitudinal motion. A higher level goal that can be described and handled by a non-communicating $\mathrm{AV}$ is the maximisation of the quality of the journey. The notion of journey's quality can be set by passengers, and may vary. Usual quality preferences can be to minimise the travel time, or to avoid some areas of the network.

The traffic controller has the overall goal of reducing congestion and minimise the overall delay for the vehicles in the network. Sensors distributed in the region provide useful information and data for UTC systems, that can act mainly by optimising traffic light stages to adapt to the current traffic conditions. On top of traditional reactive approaches such as SCOOT and SCATS, sophisticated Artificial Intelligence techniques can be exploited in this scenario, such as SURTRAC [14] or SimplifAI [15], that allow to effectively control traffic lights in a given region.

3) Infrastructural Requirements: To manage urban traffic of vehicles that are not actively communicating, conventional road traffic signal management techniques have to be exploited. Such techniques, like SCOOT and SCATS, mainly rely on surface sensors and, in order to operate, require those sensors to be in place and active. From this perspective, it is apparent that this category of communication does not impose any additional burden on traffic operators.

Satellite navigation systems are widely used as navigational aid in manually controlled vehicles [16]. In order to fully exploit connected SatNav functionalities, a working cellular network should be available. On this matter, GPRS (General Packet Radio Service) and UMTS (Universal Mobile Telecommunications System) are the most used technologies in cellular networks, at least in Europe [5].
4) Communication Requirements: Given the fact that no 2-way communication is established with other objects in the urban network, no VANETs requirements can be specified for vehicles in this category.

5) Importance of Penetration Rate: Every AV acts in isolation, without communicating with other vehicles and therefore without making assumptions on their behaviour or on the condition of the network. Penetration rate is therefore of marginal importance for this class.

\section{B. Vehicle-to-Vehicle}

$\mathrm{V} 2 \mathrm{~V}$ enables two or more vehicles to communicate and share information in real-time.

1) Knowledge and Reasoning: There are different levels of cooperation that can be implemented by exploiting V2V communications. The most intuitive is cooperative sensing: vehicles share in broadcast to all the nearby vehicles information that are collected by its onboard sensing devices. Every vehicle has then a more reliable and complete overview of the condition of the traffic and of the surroundings. Similarly, by exploiting a cascade of peer-to-peer communications, as soon as the presence of accidents or obstacles is sensed by a vehicle in a link, the information can be sent upward in the traffic stream.

Higher levels of cooperation can allow vehicles: (i) to perform cooperative lane change, where vehicles collaborate to perform safely and efficiently lane changes for vehicles in the link; (ii) to drive as a convoy, which is more common for ring roads, where vehicles in the same lane are grouped in a formation with small inter-vehicle distance; (iii) to remove the need of traffic lights by performing a cooperative intersection management [17], [18], in this scenario vehicles can negotiate to decide the order in which the junction is navigated; and (iv) in case of incidents, vehicles can cooperate to keep lanes free in order to ease the navigation of emergency response vehicles.

In all the mentioned cases, the knowledge of a single vehicle is enhanced by information collected by other nearby vehicles. From the urban network perspective, the knowledge available to each vehicle is still local, as it does not include large chunks of the urban network.

2) Achievable Goals: Despite the specific goals that correspond to the type of knowledge shared among vehicles, it is possible to identify the main goals that a V2V connected vehicle is pursuing over time: safety and comfort for passengers, improving road capacity by better exploiting lanes and junctions, increasing efficiency by identifying best speed and by cooperating for performing complex manoeuvres.

All mentioned goals are focused on the local area in which the vehicle is navigating: this is because the available knowledge only comes from onboard sensors (even though from a potentially large number of vehicles) and does not therefore allow to reason upon the wider network.

3) Infrastructural Requirements: Enacting V2V communication does not require infrastructural improvements to be implemented in the urban area. In fact, only onboard devices are needed for sensing and communicating. However, 
it is of pivotal importance that vehicles share the same communication protocol, in order to establish connections and perform communication. Producer-specific protocols should then be abandoned, in favour of more general and standardised approaches, as envisaged also by the NHTSA report [1], that allows to efficiently and reliably establish VANETs among vehicles.

4) Communication Requirements: It should be noted that the $\mathrm{V} 2 \mathrm{~V}$ architecture strongly relies on detailed local information mainly obtained by GPS. For this reason, it is fundamental that the area in which vehicles are navigating does not present aspects that can limit the GPS signal, such as tunnels, very high buildings, etc.

$\mathrm{V} 2 \mathrm{~V}$ poses serious challenges in terms of requirements of the communication. To guarantee the safety of passengers, the quality of the surroundings map, and the reliability of the negotiations for cooperative manoeuvring, it is pivotal to maintain high-frequency exchange of up-to-date and reliable data. Taking as an example the exploitation of $\mathrm{V} 2 \mathrm{~V}$ for cooperative junction management, vehicles have to exchange data almost continuously to ensure that the negotiations are successful, and to monitor the condition of the junction itself. Such a huge amount of data has to be delivered timely to each involved vehicle, and must be correct and complete. This stream of data also results in a significant computational burden on vehicles, that need to be able to received and send data at high frequency and, in the meanwhile, perform complex computation in order to reason upon received data, and to perform complex negotiations. The huge amount of data can usually also reach vehicles which are not interested in the junction, such as vehicles that have just left the junction in object, or vehicles that are in the surroundings but will take a different route. Those vehicles are still affected by the stream of data, in the sense that their onboard communication devices will receive packages and need to distinguish those which are relevant for the vehicle.

Finally, V2V communications may lead to security issues: information shared by a vehicle include a very accurate summary of its position, sensing capabilities, internal state, manufacturer, etc. Such data may be exploited for performing malicious attacks to the vehicle, or to the set of vehicles that are cooperating.

5) Importance of Penetration Rate: A high penetration rate is crucial to allow any sort of cooperation between vehicles exploiting V2V communications. In every depicted scenario, nearby vehicles need to be able to communicate in order to coordinate and to share information. In fact, in scenarios like cooperative lane change and junction management, a penetration rate lower than $100 \%$ may not allow to gain any actual benefit from V2V. For vehicles exploiting cooperative sensing, penetration rate is less critical, but the presence of legacy vehicles would dramatically reduce the spread of data among vehicles in the same lane.

\section{C. $V 2 X$}

$\mathrm{V} 2 \mathrm{X}$ communication encompasses $\mathrm{V} 2 \mathrm{~V}$ by allowing vehicles to interact also with objects of the urban network infrastructure.

1) Knowledge and Reasoning: On top of the knowledge made available by the $\mathrm{V} 2 \mathrm{~V}$ communication among vehicles, $\mathrm{V} 2 \mathrm{X}$ would allow a vehicle to gain information about a wider spectrum of cases. Vehicles can communicate with objects such as traffic lights and local sensors, and potentially cooperate. Information obtained by infrastructural object may include the level of congestion of links or junctions, the expected settings of traffic light stages, the queue, ongoing roadworks, etc.

As for the V2V case, the knowledge available to vehicles is therefore still local, but is complemented by data available to the infrastructure that is not easy to collect by vehicles. Better reasoning can then be performed in order to maximise the exploitation of data, and to improve the cooperation between vehicles.

However, in V2X is important to take into account also the infrastructure perspective. Objects, such as aforementioned traffic lights, can obtain information by communicating with vehicles, for instance about speed, directions, etc., and can then take better informed decisions when performing their tasks. Furthermore, the local infrastructure can influence the behaviour of vehicles, according to its own model of the local traffic conditions.

2) Achievable Goals: Similarly to the V2V case, main goals are set from the vehicles' viewpoint. Goals are focused on improving the quality of the journey for passengers, and to increase the road capacity by better exploiting lanes, junctions, and by optimising vehicles' speed through cooperation. However, in some sense, the local infrastructure can affect the behaviour of AVs, and is thus pursuing the general goal of reducing traffic congestion via a decentralised approach. Traffic lights can inform vehicles of high level of congestions or of growing queues, and vehicles may decide to change the route. In this case, the local infrastructure is (somehow implicitly) controlling traffic congestion, but this can also be done explicitly. Traffic lights can reason upon data sent by vehicles, and can optimise stages in order to deal with current or expected congestion. However, as the reasoning is performed at a local level, objects can not cooperate at a regional level: it may be the case then that the optimisation performed on a junction, negatively affects neighbourhood areas.

3) Infrastructural Requirements: V2X communications require a significant effort in terms of infrastructure. Objects need to be equipped with transmitting devices and with an adequate processing unit and data storing and processing capabilities. This implies an initial high economic cost for the local authorities, that have to improve the existing network infrastructure, and frequent maintenance check and substitutions.

Suitable communication protocols, and suitable monitoring techniques have also to be designed and deployed for the infrastructural objects. Traffic lights, for instance, are in the need of identifying vehicles which are approaching the controlled junction and being able to communicate with them. At the same time, the infrastructure does not need 
to receive all the message exchanged by vehicles in $\mathrm{V} 2 \mathrm{~V}$ communications.

4) Communication Requirements: In terms of communication requirements, $\mathrm{V} 2 \mathrm{~V}$ and $\mathrm{V} 2 \mathrm{X}$ are similar. In both cases high-frequency up-to-date and reliable data need to be shared between vehicles and objects. This is crucial for cooperation between vehicles, but it can be somehow relaxed in the cooperation and communication between vehicles and infrastructural objects. Let us take traffic lights as an example: while effective and high-speed communication is fundamental to maximise the benefit of V2X, traffic lights are physical artefacts; this means that even in the case of a lack of communication or corrupted data, traffic lights will still be in the position of controlling the traffic of the junction. Given this angle, $\mathrm{V} 2 \mathrm{X}$ is more robust -in the sense that vehicles do not have to blindly rely on information communicated.

5) Importance of Penetration Rate: Penetration rate differently affects the V2V and the V2X capabilities. With regards to the $\mathrm{V} 2 \mathrm{~V}$ class, as observed in the previous sections, it is crucial an extremely high penetration rate in order to gain any benefit by the cooperation and communication. Some benefits of V2X can be obtained by vehicles in cases where the penetration rate is low. A vehicle can still communicate with traffic lights in order to optimise its speed according to the expected queue and green time, and may still gain information about congestion on the other side of the junction. Clearly, to maximise benefits for AVs, a high penetration rate is beneficial so that vehicles can actively cooperate reduce queues and waiting times.

From the infrastructure perspective, a higher penetration rate leads to more and reliable data being available to the object to perform its task and pursue its goals. In cases where the penetration rate is low, no significant benefits would be observed by the infrastructure, as it will have to rely only on existing sensors.

\section{I2V and V2I}

The I2V class covers approaches where AVs can establish a 2-way communication with a centralised urban traffic control centre of the region. In order to focus the analysis on this specific kind of interaction, we assume that vehicles are not performing additional $\mathrm{V} 2 \mathrm{~V}$ or $\mathrm{V} 2 \mathrm{X}$ communications, or that, whether such other communications are performed, they do not affect the overall behaviour of the vehicle with regards to the route to follow for navigating the network.

1) Knowledge and Reasoning: The traffic control centre holds knowledge about the whole controlled region -via sensors on the surface-, and receives information from AVs about their actual position, their intentions, and preferences on the route to follow or on waypoints. The control centre is therefore in the best position to reason with global information and to provide instructions -personalised according to the requirements of passengers- to vehicles. Data from different available sources (such as sensors, cameras, etc.) can be linked with data received from AVs, in order to provide rich information to the reasoning system. In terms of reasoning upon available data, AI approaches seem to be the more appropriate for dealing with the volume of data, and the complexity of the tasks to perform.

On the contrary, limited knowledge is made available to vehicles. They would not obtain a complete overview of the status of the urban network, but are given optimal routes by the control centre. Additionally, they may be allowed to receive an explanation of the selected route. In that sense, $\mathrm{I} 2 \mathrm{~V}$ shifts knowledge and reasoning on the control centre, leaving vehicles to deal with only the actual driving tasks.

2) Achievable Goals: On the traffic control side, this communication class allows the implementation and maintenance of region-wide high level goals: air quality, congestion, planning for events and road works, etc. The centralised approach can provide intelligence and adaptability to make the transport network more resilient to exceptional or unexpected events, as well as increasing its capacity and efficiency.

Remarkably, this class of communication can also directly support vehicle sharing. Given the destination of a vehicle, and the location of passengers keen to ride a vehicle in the same direction, the traffic control centre can calculate the most suitable route to accommodate the largest number of requests. It is also possible to coordinate $\mathrm{AVs}$ movement with public transport, to support a better integration.

It is out of the scope of this class, and computationally infeasible, to require the control centre to perform any sort of local (with regards to a specific vehicle) goal. However, preferences of passengers of a vehicle with regards to the route to set can be communicated to the control centre, that is in the position to try to accommodate them.

3) Infrastructural Requirements: Significant support has to be put in place in order to allow the communication between vehicles in the region and the centralised UTC architecture. Available cellular networks, or specialised VANETs can be exploited for exchanging information between AVs and the centralised traffic control centre of the urban region.

The volume of data that the traffic control centre has to deal with, and the complexity of the reasoning to be performed, poses some serious challenges to the centralised structure. It must be able to store and analyse received data, to monitor the condition of the network and to predict potential future issues, and to quickly generate suitable routes for AVs in order to achieve the defined goals.

4) Communication Requirements: The strict communication requirements of $\mathrm{V} 2 \mathrm{~V}$ and $\mathrm{V} 2 \mathrm{X}$ can be relaxed. In the I2V/V2I scenarios the communication can, to some extent, allow a higher latency than in the previously described categories. This is because the aims of the communication are different: the control centre does not need to provide a complete view of the network or of the surrounding conditions to the vehicle to control movements or manoeuvres. The control centre provides routes to follow for reach the destination, and may add information about traffic or explanations. Therefore, the I2V communication will be much easier and faster and, the fact some data is missing or some information arrive late does not affect the safety of vehicles.

According to the level of service required by vehicles, private information may not be disclosed. As a minimum, 
the traffic control centre needs the accurate GPS position and the desired destination of the AV. Other information may be useful for better services, such as optimising routes according to passengers' preferences.

5) Importance of Penetration Rate: To some extent, in I2V/V2I the penetration rate is not extremely important. Information about current traffic conditions are already available for traffic controllers via surface sensors, and can be exploited for routing AVs which are capable of communicate using I2V. However, a high penetration rate increases the impact of the traffic control centre strategies, allows a better support for higher level of services, and reduces the unpredictability of vehicles' behaviour and of future congestions.

\begin{tabular}{|l|l|l|l|l|}
\hline & No comm & V2V & V2X & I2V \& V2I \\
\hline Level of deliberation & low & med & med & high \\
Infrastructure Costs & none & low & med & high \\
Running Costs & none & low & med & high \\
Penetration Rate & none & high & low & med \\
\hline
\end{tabular}

TABLE I

SUMMARY OF IMPORTANT FACTORS OF THE DIFFERENT COMMUNICATION TYPES.

\section{DiscuSSION AND CONCLUSIONS}

In this work, we provided a principled and detailed analysis of the impact of vehicular communication on the reasoning capabilities of $\mathrm{AVs}$ and, to some extent, of the urban traffic control. A summary of the most important factors is shown in Table I. The level of deliberation refers to the availability of knowledge for reasoning at a global network level with longer term goals (high), or at a very localised level with shorter term goals (low). Costs can be divided in infrastructure costs, that correspond to the upfront costs due to the installation of additional devices on the network surface, and running costs -that have to be faced by transport authorities in order to sustain the corresponding approach. Finally, the penetration rate shows how important is the penetration rate with regards to the potential benefits. In a nutshell, according to the performed analysis, very significant region-wide benefits can be gained by exploiting I2V and V2I communications, but this comes with significant upfront and maintenance costs. Hence, I2V and V2I communications might be beneficial in dense urban areas such as city centres where intelligent decision making is crucial for maintaining reasonable quality of the journey. The other types of communications allow more localised benefits while posing limited pressure on traffic agencies funds. So they can be exploited in less "demanding" parts of the road network.

Future work will include the assessment for each communication class, of how functionalities can be provided by different implementations and deployments, and what additional limitations may arise. We also plan to analyse how different types of vehicular communications can be fruitfully combined.

\section{ACKNOWLEDGEMENT}

Mauro Vallati was partially supported by the EPSRC grant EP/R51343X/1 (AI4ME). Lukáš Chrpa was funded by the Czech Science Foundation (project no. 17-17125Y).

\section{REFERENCES}

[1] J. Harding, G. Powell, R. Yoon, J. Fikentscher, C. Doyle, D. Sade, M. Lukuc, J. Simons, and J. Wang, "Vehicle-to-vehicle communications: Readiness of v2v technology for application,” Tech. Rep., 2014.

[2] C. Wei, "V2x communication in europe from research projects towards standardization and field testing of vehicle communication technology," Computer Networks, vol. 55, no. 14, pp. 3103 - 3119, 2011.

[3] L. Hobert, A. Festag, I. Llatser, L. Altomare, F. Visintainer, and A. Kovacs, "Enhancements of v2x communication in support of cooperative autonomous driving," IEEE Communications Magazine, vol. 53, no. 12, pp. 64-70, 2015.

[4] S. Djahel, N. Jabeur, R. Barrett, and J. Murphy, "Toward v2i communication technology-based solution for reducing road traffic congestion in smart cities," in Networks, computers and communications (ISNCC), 2015 international symposium on. IEEE, 2015, pp. 1-6.

[5] J. Santa, A. F. Gómez-Skarmeta, and M. Sánchez-Artigas, "Architecture and evaluation of a unified $\mathrm{v} 2 \mathrm{v}$ and $\mathrm{v} 2 \mathrm{i}$ communication system based on cellular networks," Computer Communications, vol. 31, no. 12, pp. 2850-2861, 2008.

[6] United Nations, "World urbanization prospect," http://esa.un.org/unpd/wup/Publications/Files/WUP2014Highlights.pdf, 2014, accessed: 2017-11-02.

[7] J. C. Falcocchio and H. S. Levinson, "The costs and other consequences of traffic congestion," in Road Traffic Congestion: A Concise Guide. Springer, 2015, pp. 159-182.

[8] R. Bretherton, "Scoot urban traffic control system: Philosophy and evaluation," in Proceedings of the 6th IFAC/IFIP/IFORS Symposium on Control, Computers, and Communications in Transportation, 1989, pp. 237-239.

[9] R. Vincent and J. Pierce, "Self-optimising signal control for isolated intersections," in Crowthorne: Transport and Road Research Laboratory Research Report, no. 170, 1988.

[10] P. Lowrie, "The sydney coordinated adaptive traffic system-principles, methodology, algorithms," in Proceedings of the International Conference on Road Traffic Signalling, no. 207, 1982.

[11] C. Urmson, J. Anhalt, D. Bagnell, C. Baker, R. Bittner, M. Clark, J. Dolan, D. Duggins, T. Galatali, C. Geyer, et al., "Autonomous driving in urban environments: Boss and the urban challenge," Journal of Field Robotics, vol. 25, no. 8, pp. 425-466, 2008.

[12] G. De Nunzio, C. C. Wit, P. Moulin, and D. Di Domenico, "Ecodriving in urban traffic networks using traffic signals information," International Journal of Robust and Nonlinear Control, vol. 26, no. 6, pp. 1307-1324, 2016.

[13] D. Beattie, L. Baillie, M. Halvey, and R. McCall, "Adapting satnav to meet the demands of future automated vehicles," in CHI 2015 Workshop on Experiencing Autonomous Vehicles: Crossing the Boundaries between a Drive and a Ride, 2015.

[14] X.-F. Xie, S. Smith, and G. Barlow, "Schedule-driven coordination for real-time traffic network control," in Proceedings 22nd International Conference on Automated Planning and Scheduling (ICAPS), 2012.

[15] T. L. McCluskey and M. Vallati, "Embedding automated planning within urban traffic management operations," in Proceedings of the Twenty-Seventh International Conference on Automated Planning and Scheduling (ICAPS), 2017, pp. 391-399.

[16] L. Meng and T. Reichenbacher, "Map-based mobile services," in Mapbased Mobile Services. Springer, 2005, pp. 1-10.

[17] X. Qian, J. Gregoire, F. Moutarde, and A. De La Fortelle, "Prioritybased coordination of autonomous and legacy vehicles at intersection," in Intelligent Transportation Systems (ITSC), 2014 IEEE 17th International Conference on, 2014, pp. 1166-1171.

[18] M. Ferreira, R. Fernandes, H. Conceição, W. Viriyasitavat, and O. K. Tonguz, "Self-organized traffic control," in Proceedings of the seventh ACM international workshop on VehiculAr InterNETworking, 2010, pp. 85-90. 